\title{
Digitale Medien
}

\author{
Katrin Tiidenberg: Selfies: Why We Love (and Hate) Them \\ Bingley: Emerald Publishing Limited 2018, 145 S., \\ ISBN 9781787437173, EUR 22,-
}

Katrin Tiidenberg stellt in ihrem Buch Selfies: Why We Love (and Hate) Them auf verständliche und engagierte Weise die aktuelle Forschung zu Selfies vor. Die vier Kapitel sind mit Fragen überschrieben: „What are Selfies?", „How Do We Selfie?“, „(Why) Do Selfies Matter?" und „Post-Selfie?“. Tiidenbergs Interesse ist es, kulturelle Werte und soziale Normen zu untersuchen, die sich in der Praxis des Selfies manifestieren. Die Medienwissenschaftlerin erforscht seit 2011, wie sich Identität und Beziehungen in bildzentrierten sozialen Medien wie Tumblr und Instagram formen und ausdrücken. Selfies befänden sich dabei in Ambivalenz: Einerseits zögen sie viel Aufmerksamkeit auf sich, andererseits würden sie als dieser Aufmerksamkeit nicht wert betrachtet, da sie überhöhten Selbstbezug und mangelnden künstlerischen Wert aufwiesen (vgl. S.6). Tiidenberg argumentiert, dass Selfies im jeweiligen Kontext betrachtet werden müssten: Wie andere kulturelle Praktiken auch, sind sie nicht einfach, gut' oder ,schlecht', sondern vielgestaltig - oder: vielgesichtig (vgl. S.8): „To understand how we do selfies we have to think about what the popular ways of making sense of selfies are today, what the ruling norms say about appearances, about who gets to share selfies, and who gets told to be ashamed of their desire to be seen? What are the accepted ways of being seen as a mother, a man, a teacher or a soldier?" (S.58).

Ein Selfie beinhaltet visuelle, fotografische und vernetzte Selbst-Repräsentationen (vgl. S.18), die simultan stattfinden. Tiidenbergs Definition besagt, dass eine solche Selbst-Repräsentation nicht unbedingt das Gesicht zeigen müsse - sie bezieht sich hier auf so genannte „sexy selfies“ (S.21), die bewusst nur Körper(teile) zeigten. Die Autorin führt aus, dass vernetzte Bilder nicht geteilt werden müssen; sie müssen lediglich potenziell verschickbar sein (S.22). Nach historischer, archäologischer und kunstgeschichtlicher Kontextualisierung des menschlichen Interesses an Darstellung und Selbstporträts widmet sich Tiidenberg dem fotografischen Selbstporträt und dem Einfluss der digitalen und Smartphone-Fotografie (vgl. S.27ff.). Um sich dem Phänomen Selfie zu nähern, greift Tiidenberg auf andere vernetzte Selbstdarstellungen zurück, wie Profilbilder und Statusupdates bei Twitter oder Facebook (vgl. S.36ff.). Die Praxis des Selfies formt Tiidenberg als neuen Ausdruck: ,Doing Selfie؛. Die Aktivitäten, die in eigenen Selfies abgebildet sind, wurden durch das Betrachten vorliegender Selfies inspi- 
riert: „The situation of doing a selfie is dense with memories, experiences and expectations" (S.49). Tiidenberg zitiert aus ihren ethnografischen Studien mit Frauen, die ,sexy selfies' auf Tumblr posten, sowie mit ihren Studierenden, die über ihre Ambivalenz berichten, Probe-Selfies auf ihren Smartphones zu lassen: Es sei peinlich, wenn andere diese beim Scrollen finden würden (vgl. S.49). Technische Qualitäten wie Licht, Tiefenschärfe oder die Auswahl der Bilder ist individuell; dies wird als Ausdruck des eigenen Körpers und Selbst verstanden und kann in Bevorzugung bestimmter Apps münden (vgl. S.56f.). In einer von Tiidenberg untersuchten Community war es üblich, Selfies zu reposten - als eine Person ein Bild übernahm, neu beschnitt und mit einem eigenen Wasserzeichen postete, entbrannte Streit über die Möglichkeiten und Grenzen von Bildübernahme und Affirmation. Tiidenbergs Interesse an Selfies besteht darin, aufzuzeigen, warum diese starke Emotionen hervorrufen (vgl. S.75ff.). Als Beispiele nennt die Autorin „funeral selfies" (S.60f.), die zunächst abgelehnt wurden, nach Aussagen von Wissenschaftler_innen aber eine Funktion des Trauerns haben können, sowie das Large Labia Project, in dem Frauen Schamlippen-Selfies posteten, um gegen Stigmatisierung und Normierung der Vulva zu protestieren (vgl. S.64).
Tiidenbergs Fokus liegt auf Beispielen, die Empowerment durch Selfies zeigen. Dies kann durch positivere Selbstwahrnehmung geschehen, aber auch durch Reposten von Beiträgen anderer, deren Urheber_innen dadurch mehr Selbstvertrauen geschenkt werden soll (vgl. S.67, S.69, S.89ff. und S.93f.). Tiidenberg erklärt starke negative Gefühle gegenüber Selfies damit, dass hier lange gültige soziale Normen gebrochen werden (vgl. S.87f.). Beispielsweise schreibe sich die männliche Dominanz in Bildern fort und entstehe damit die Zuschreibung, Männer seien einer Abbildung mehr wert als Frauen: „The judgment that young women's selfie practices are narcissistic could be seen as an underlying anxiety about them suddenly bypassing the traditional gatekeepers of visibility" (S.81). Katrin Tiidenberg schließt ihr Buch mit generellen Überlegungen zur Internet- und Plattformgesellschaft sowie einer dezidierten Aufforderung zu individueller Ethik: „Critical literacy in this case means developing our own sensibility for nuance, our own tolerance for the shades of gray and our empathy and solidarity towards other users of social media platforms whose needs and ability to effect change may be different than ours" (S.130). Und genau dabei, so Tiidenbergs Fazit, können Selfies helfen.

Evelyn Runge (Jerusalem) 